\title{
Ultrasonic imaging characteristics of transplanted kidneys with delayed graft function
}

\author{
W.X. Liang' ${ }^{1}$, M.J. Cai ${ }^{2}$, L. Jiang ${ }^{1}$, Y.Q. Xie ${ }^{1}$, W.L. Yuan ${ }^{1}$ and H. Zhang ${ }^{1}$ \\ ${ }^{1}$ Department of Ultrasound Medicine, \\ The Third Affiliated Hospital of Guangzhou Medical University, \\ Guangzhou, China \\ ${ }^{2}$ Department of Radiology, \\ The Third Affiliated Hospital of Guangzhou Medical University, \\ Guangzhou, China \\ Corresponding author: W. Liang \\ E-mail: weixiangliang89@sina.cn
}

Genet. Mol. Res. 13 (3): 6878-6884 (2014)

Received July 10, 2013

Accepted May 16, 2014

Published August 29, 2014

DOI http://dx.doi.org/10.4238/2014.August.29.9

\begin{abstract}
We investigated the ultrasonic imaging characteristics of transplanted kidneys with delayed graft function (DGF). Ultrasonography was performed in 44 patients after kidney transplantation, and a time-intensity analysis was performed to compare the differences between patients with normal graft function (NGF) and those with DGF. Compared with the NGF group, the DGF group had earlier arrival time, shorter time to peak, and higher arrival intensity and peak intensity $(\mathrm{P}<0.05)$. The variation-ofintensity parameters in different renal cortices increased, whereas the variation-of-time parameter decreased, in those with DGF $(\mathrm{P}<0.05)$. In conclusion, compared with the NGF group, the microcirculation perfusion of transplanted kidneys in the DGF group showed higher perfusion with earlier arrival time, shorter time to peak, and higher arrival intensity and peak intensity. In addition, the
\end{abstract}


intensity variations of contrast agent in different renal cortices from patients with DGF were greater, whereas the variations in perfusion time were smaller than those in patients with NGF.

Key words: Ultrasonography; Kidney transplantation; Delayed graft function; Time-intensity curve

\section{INTRODUCTION}

Delayed graft function (DGF) is one of the most common complications after kidney transplantation. Previous studies have indicated that DGF incidence ranges from $4 \%$ to $10 \%$ in patients receiving a live graft (Perico et al., 2004) and from 5 to $50 \%$ in patients receiving a cadaver graft (Ojo et al., 1997; Sellers et al., 2000; Gjertson, 2000). Factors that can increase the risk for DGF include local ischemia due to long-term freezing and rewarming, an older donor, brain death or abnormal cardiovascular hemodynamics of the donor before kidney removal, a healthier recipient, and the use of drugs toxic to the kidney (Shoskes and Halloran, 1996; Ojo et al., 1997). The incidence of DGF in patients receiving a graft from a donor with cardiovascular disease is $20 \%$ higher than that in those receiving a graft from a healthy donor (Sola et al., 2004). Because of the wide range of possible contributing factors, there is still no effective way to prevent DGF.

With compensation, renal function in patients with DGF after kidney transplantation can remain stable in the short term. However, previous studies have indicated that DGF can promote acute rejection, which is closely correlated with decreased graft survival during the late phase, and is also an independent risk factor for cytomegalovirus infection and hypertension after kidney transplantation (Sola et al., 2004; Tapiawala et al., 2010). Therefore, early diagnosis and treatment of DGF is critical for protecting graft function. Serum creatinine level and glomerular filtration rate are the most commonly used indicators of transplanted kidney function in clinical practice. However, studies suggested that these parameters do not accurately reflect transplanted kidney function, and that there is no precise index available for clinical evaluation (Brier et al., 2003; Marcén et al., 2010). Therefore, it is critical to seek methods for early prediction and treatment of DGF. Contrast-enhanced ultrasonography (CEUS) is a novel non-invasive method for evaluating organ microcirculation (Correas et al., 2006; Barozzi et al., 2010), and has the potential to provide unique advantages in the early diagnosis of DGF. Our study investigated the potential utility of CEUS in diagnosing DGF by comparing CEUS differences between patients with DGF and those with normal graft function (NGF). We also investigated the use of CEUS for providing reference values for the clinical diagnosis and treatment of DGF.

\section{MATERIAL AND METHODS}

This study was supported by The Third Affiliated Hospital of Guangzhou Medical University; the study protocol and ethics were approved by this institution. Informed consent was provided by all patients participating in the study. Subjects were patients undergoing allograft kidney transplantation between May 2010 and May 2012. A diasonograph (GE LogiQ 9) and a broadband $4 \mathrm{C}$ convex array probe equipped with CEUS technology and time-intensi- 
ty curve (TIC) software were used. On the fifth day after kidney transplantation, patients who received an allograft underwent CEUS examination with a probe with a mechanical index of 0.08-0.13 after receiving a bolus injection of $1.2 \mathrm{~mL}$ contrast agent (SonoVue) into the superficial vein of the right forearm. Maximal section imaging with full observation of the renal cortex was recorded for TIC analysis.

Two regions of interest (ROI) with stable images in the same cortex area with a frame of $10 \times 10 \mathrm{~mm}$ were selected and quantitatively analyzed independently by 2 radiologists. The means of the DGF and NGF groups were used for statistical analysis. TIC analysis values included: arrival time $\left(\mathrm{AT}_{1}, \mathrm{AT}_{2}\right)$, time to peak $\left(\mathrm{TTP}_{1}, \mathrm{TTP}_{2}\right)$, absolute TTP $\left(\mathrm{ATTP}_{1}, \mathrm{ATTP}_{2}\right)$, arrival intensity $\left(\mathrm{AI}_{1}, \mathrm{AI}_{2}\right)$, peak intensity $\left(\mathrm{PI}_{1}, \mathrm{PI}_{2}\right)$, terminal intensity $\left(\mathrm{TI}_{1}, \mathrm{TI}_{2}\right)$, area under the curve $\left(\mathrm{AUC}_{1}, \mathrm{AUC}_{2}\right)$, and differential indices of the 2 ROIs.

Clinical and biochemical examinations were performed for 3 consecutive weeks to assign the recruited subjects into 2 groups. Criteria for inclusion in the DGF group included: 1) early-stage postoperative oliguria, anuria, or diuresis with a subsequent sudden drop of urine volume and recovery to normal within days or weeks; 2) slow decrease or sudden increase of serum creatinine level in the absence of a decrease to $400 \mu \mathrm{mol} / \mathrm{L}$ within 1 week of surgery; 3 ) gradual increase of serum potassium level with a slow increase or obvious decrease of hemoglobin; and 4) complications such as hypotension, hypertension, edema, or chest congestion.

Criteria for inclusion in the NGF group included: 1) gradual recovery of urine volume to normal range within 1 week of surgery (i.e., daily urine volume between 1500 and 2000 $\mathrm{mL})$; 2) no complications such as hyperthermia, oliguria, graft swelling, tenderness, or hypertension; and 3) no acute tubular necrosis of graft or toxic drug nephropathy.

Continuous variables are reported as means $\pm \mathrm{SD}$, and categorical variables are expressed as a rate or percentage. Differences between the 2 groups were compared using the SPSS17.0 software, with the independent $t$-test for continuous variables and the $\chi^{2}$ test for categorical variables. $\mathrm{P}<0.05$ was the threshold for significance.

\section{RESULTS}

A total of 44 patients ( 29 men and 15 women) with a mean age of $44.23 \pm 14.89$ years were recruited. There were no significant differences between the DGF group $(\mathrm{N}=20)$ and the NGF group $(\mathrm{N}=24)($ Table 1$)$.

Table 1. General comparison of characteristics of the delayed (DGP) and normal graft function (NGF) groups.

\begin{tabular}{lcccc}
\hline & DGF group & NGF group & $t / \chi^{2}$ value & P \\
\hline Number & 20 & 24 & - & - \\
Age (years) & $43.90 \pm 16.26$ & $44.50 \pm 14.00$ & -0.132 & 0.896 \\
Gender (male/female) & $13 / 7$ & $16 / 8$ & 0.013 & 1.000 \\
\hline
\end{tabular}

$\mathrm{DGF}=$ delayed graft function; NGF $=$ normal graft function.

\section{Time indices comparison}

$\mathrm{AT}_{1}, \mathrm{TTP}_{1}, \mathrm{ATTP}_{1}, \mathrm{TTP}_{2}$ and $\mathrm{ATTP}_{2}$ values in the DGF group were $10.59 \pm 2.22 \mathrm{~s}$, $20.84 \pm 2.61 \mathrm{~s}, 10.26 \pm 2.93 \mathrm{~s}, 20.59 \pm 3.26 \mathrm{~s}$, and $9.91 \pm 3.29 \mathrm{~s}$, respectively. These values were significantly shorter than those in the NGF group $(12.67 \pm 4.11 \mathrm{~s}, 25.96 \pm 7.95 \mathrm{~s}, 13.23$ 
$\pm 7.62 \mathrm{~s}, 26.24 \pm 8.04 \mathrm{~s}$, and $14.06 \pm 7.51 \mathrm{~s}$, respectively $)(\mathrm{P}<0.05)$. This indicated that the AT of the contrast agent into the graft and the TTP in the DGF group were quicker than those in the NGF group. There was no significant difference in $\mathrm{AT}_{2}$ between the 2 groups (Table 2).

Table 2. Time indices comparison of the delayed (DGF) and normal graft function (NGF) groups.

\begin{tabular}{lcccc}
\hline & DGF group & NGF group & $t / \chi^{2}$ value & P \\
\hline $\mathrm{N}$ & 20 & 24 & - & - \\
Age (years) & $43.90 \pm 16.26$ & $44.50 \pm 14.00$ & -0.132 & 0.896 \\
Gender (male/female) & $13 / 7$ & $16 / 8$ & 0.013 & 1.000 \\
$\operatorname{AT}_{1}(\mathrm{~s})$ & $10.59 \pm 2.22$ & $12.67 \pm 4.11$ & -2.030 & 0.049 \\
$\operatorname{TTP}_{1}$ (s) & $20.84 \pm 2.61$ & $25.96 \pm 7.95$ & -2.970 & 0.006 \\
$\operatorname{ATTP}_{1}()$ & $10.26 \pm 2.93$ & $13.23 \pm 7.62$ & -1.800 & 0.082 \\
$\operatorname{AT}_{2}$ (s) & $10.69 \pm 2.27$ & $12.18 \pm 3.85$ & -1.597 & 0.118 \\
$\operatorname{TTP}_{2}$ (s) & $20.59 \pm 3.26$ & $26.24 \pm 8.04$ & -3.147 & 0.004 \\
$\operatorname{ATTP}_{2}$ (s) & $9.91 \pm 3.29$ & $14.06 \pm 7.51$ & -2.444 & 0.020 \\
\hline
\end{tabular}

$\mathrm{AT}=$ arrival time; $\mathrm{TTP}=$ time to peak; $\mathrm{ATTP}=$ absolute time to peak $\mathrm{DGF}=$ delayed graft function; $\mathrm{NGF}=$ normal graft function.

\section{Intensity indices comparison}

The $\mathrm{AI}_{1}, \mathrm{AI}_{2}$, and $\mathrm{PI}_{1}$ values in the DGF group were $-71.57 \pm 4.05,-73.34 \pm 3.18$, and $-47.06 \pm 4.39 \mathrm{~dB}$, respectively; these values were higher than those in the NGF group (-75.02 $\pm 1.54,-75.69 \pm 1.55$, and $-51.57 \pm 2.99 \mathrm{~dB}$, respectively). The differences were significant $(\mathrm{P}$ $<0.05)$. There were no significant differences in $\mathrm{PI}_{2}, \mathrm{TI}_{1}, \mathrm{TI}_{2}, \mathrm{AUC}_{1}$, or $\mathrm{AUC}_{2}$ values between the 2 groups (Table 3 ).

Table 3. Intensity indices comparison of the delayed (DGF) and normal graft function (NGF) groups.

\begin{tabular}{lllrr}
\hline & DGF group & NGF group & $t$ value & \multicolumn{1}{c}{ P } \\
\hline $\mathrm{AI}_{1}(\mathrm{~dB})$ & $-71.57 \pm 4.05$ & $-75.02 \pm 1.54$ & 3.595 & 0.001 \\
$\mathrm{PI}_{1}(\mathrm{~dB})$ & $-47.06 \pm 4.39$ & $-51.57 \pm 2.99$ & 4.031 & 0.000 \\
$\mathrm{TI}_{1}(\mathrm{~dB})$ & $-57.67 \pm 2.79$ & $-58.31 \pm 3.43$ & 0.669 & 0.507 \\
$\mathrm{AUC}_{1}$ & $1741.8 \pm 326.89$ & $1839.4 \pm 437.54$ & -0.760 & 0.452 \\
$\mathrm{AI}_{2}(\mathrm{~dB})$ & $-73.34 \pm 3.18$ & $-75.69 \pm 1.55$ & 3.019 & 0.006 \\
$\mathrm{PI}_{2}(\mathrm{~dB})$ & $-49.19 \pm 3.61$ & $-51.14 \pm 2.99$ & -958 & 0.057 \\
$\mathrm{TI}_{2}(\mathrm{~dB})$ & $-58.84 \pm 4.15$ & $-58.42 \pm 2.74$ & -0.392 & 0.698 \\
$\mathrm{AUC}_{2}$ & $1755.4 \pm 321.13$ & $1873.3 \pm 410.48$ & -0.967 & 0.340 \\
\hline
\end{tabular}

$\mathrm{AI}=$ arrival intensity; $\mathrm{PI}=$ peak intensity; $\mathrm{TI}=$ terminal intensity; $\mathrm{AUC}=$ area under the curve; $\mathrm{DGF}=$ delayed graft function; NGF = normal graft function.

\section{Analysis of TIC variation}

The AT and ATTP variation of the 2 ROIs in the DGF group were $0.19 \pm 0.10 \mathrm{~s}$ and $0.95 \pm 0.65 \mathrm{~s}$, respectively; these values were significantly smaller than those in the NGF group $(0.74 \pm 0.65$ and $1.54 \pm 1.08 \mathrm{~s}$, respectively) $(\mathrm{P}<0.05)$. The differential AI and PI values of the 2 ROIs in the DGF group were $2.14 \pm 1.13$ and $2.63 \pm 2.18 \mathrm{~dB}$, respectively; these values were significantly larger than those in the NGF group $(0.79 \pm 0.57$ and $1.24 \pm 0.77 \mathrm{~dB}$, respectively) $(\mathrm{P}<0.01)$. These results suggest that the perfusion time variation of contrast agent in the renal cortex of the DGF group was smaller than that of the NGF group, whereas 
the perfusion intensity variation of contrast agent in the renal cortex of the DGF group was larger than that of the NGF group. There were no significant differences in $\mathrm{TTP}_{1,2}, \mathrm{TI}_{1,2}$, or $\mathrm{AUC}_{1,2}$ between the 2 groups (Table 4).

Table 4. Variation analysis of the time-intensity curve in the delayed (DGF) and normal graft function (NGF)
groups.
\begin{tabular}{lcccc} 
& & \\
& DGF group & NGF group & $t$ value & P \\
\hline $\mathrm{AT}_{1,2}(\mathrm{~s})$ & $0.19 \pm 0.10$ & $0.74 \pm 0.65$ & -4.024 & 0.000 \\
$\mathrm{TTP}_{1,2}(\mathrm{~s})$ & $0.85 \pm 0.57$ & $1.35 \pm 1.18$ & -1.853 & 0.073 \\
$\mathrm{ATTP}_{1,2}(\mathrm{~s})$ & $0.95 \pm 0.65$ & $1.54 \pm 1.08$ & -2.240 & 0.031 \\
$\mathrm{AI}_{1,2}(\mathrm{~dB})$ & $2.14 \pm 1.13$ & $0.79 \pm 0.57$ & 4.833 & 0.000 \\
$\mathrm{PT}_{1,2}(\mathrm{~dB})$ & $2.63 \pm 2.18$ & $1.24 \pm 0.77$ & 2.911 & 0.006 \\
$\mathrm{TI}_{1,2}(\mathrm{~dB})$ & $1.89 \pm 0.59$ & $1.67 \pm 0.98$ & 0.896 & 0.376 \\
$\mathrm{AUC}_{1,2}$ & $102.67 \pm 152.45$ & $130.8 \pm 76.94$ & -0.793 & 0.460 \\
\hline
\end{tabular}

$\mathrm{AT}=$ arrival time; $\mathrm{TTP}=$ time to peak; $\mathrm{ATTP}=$ absolute time to peak; $\mathrm{AI}=$ arrival intensity; $\mathrm{PI}=$ peak intensity; $\mathrm{TI}=$ terminal intensity; $\mathrm{AUC}=$ area under the curve; $\mathrm{DGF}=$ delayed graft function; $\mathrm{NGF}=$ normal graft function.

\section{DISCUSSION}

DGF manifests as the immediate loss of acute function of the transplanted kidney, and is one of the most common complications after transplantation. DGF not only complicates treatment after kidney transplantation, but also prolongs hospitalization, increases associated costs, and increases the risk of graft morbidity after surgery, acute and chronic rejection, chronic nephropathy, and function loss in the early stages. A previous study has indicated that DGF increases the incidence of graft function loss by $41 \%$ and acute rejection by $38 \%$ during the first year, and that serum creatinine levels remain high for a prolonged period (Yarlagadda et al., 2009).

The occurrence of DGF is affected by many factors; injury to the transplanted kidney resulting from ischemic reperfusion plays an important role. Using a thermal dissipation probe inserted into the cortex of the transplanted kidney, Angelescu et al. (2003) found lower microcirculatory perfusion in DGF patients than in patients with a normally functioning transplanted kidney. In addition, ischemic reperfusion has been shown to result in increased serum levels of endothelin and endothelin-1, but decreased levels of the vasodilator nitric oxide, which increases vascular resistance in the transplanted kidney (Schilling et al., 1996; Huang et al., 2002; Inman et al., 2003; Perico et al., 2004; Zlotnick et al., 2010).

CEUS is an effective non-invasive technique that uses an ultrasonic contrast agent to evaluate the microcirculation of organs and tissues. The application of CEUS in the examination of transplanted kidneys allows observation of dynamic blood perfusion and distribution in the transplanted kidney, and can allow quantitative analysis of the perfusion of the transplanted kidney through TIC analysis. Currently, CEUS is used most often in studies related to diagnosis of acute and chronic rejection after kidney transplantation, and provides guidance for clinical treatment in such cases.

There have been very few studies of the use of CEUS in kidney transplantation in patients with DGF. Early diagnosis and treatment of DGF can effectively protect the function of the transplanted kidney, improving the prognosis and reducing postsurgical complications. Although there are many methods for diagnosing DGF in the clinic, most of them that must 
be combined with clinical laboratory tests are not precise, and can take a week to complete, which can result in delayed diagnosis or misdiagnosis. Therefore, for this study, we chose the time window of 5 days after surgery to diagnose and analyze DGF, distinguish early complications such as acute rejection, renal artery thrombus and stenosis, and identify ureter obstruction. Therefore, CEUS can, in theory, evaluate microcirculatory changes in transplanted kidneys and provide a reference for targeting early clinical treatment. Unfortunately, there are few related studies providing conclusive data.

In our study, the microcirculatory perfusion in the transplanted kidneys of patients in the DGF group showed earlier AT and TTP, higher AI and TTI, and a larger variation of contrast agent intensity in different renal cortex regions of the same graft than those in patients in the NGF group. This suggests that transplanted kidneys in DGF patients have a higher rate of perfusion, which differs across different cortex regions.

The higher transplanted kidney perfusion rate may be related to the inflammatory response induced by DGF. This is consistent with the results of a previous study showing increased interleukin- 8 levels and elevated numbers of neutrophilic granulocytes in the urine of DGF patients (Yarlagadda et al., 2008). The different perfusion intensities in different regions of the renal cortex suggest uneven distribution of resistance to renal microcirculation in DGF patients, which may be related to the pathological changes of terminal vasculature induced by ischemic reperfusion in the transplanted kidney (Inman et al., 2003; Huang et al., 2002). This may also be the mechanism of difference in the study by Angelescu et al. (2003) (Figure 1).

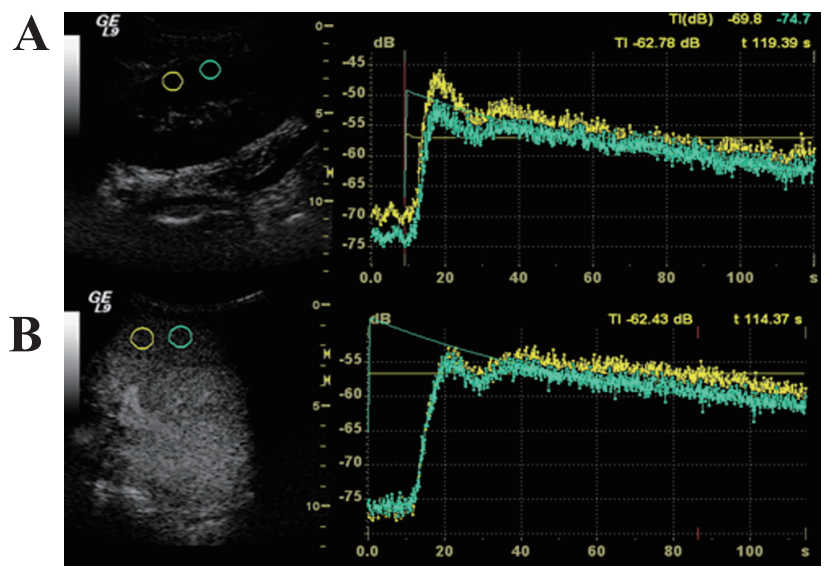

Figure 1. A. Time-intensity curve of transplanted kidney with delayed graft function, demonstrating earlier arrival time and shorter time to peak, higher arrival intensity and peak intensity, larger intensity variation, and smaller time variation in different regions of the renal cortex; B. time-intensity curve of transplanted kidney with normal function.

In addition, our study indicates that the perfusion time variation is smaller in different renal cortices in DGF patients, which may be related to the dilation of small and medium-sized arteries; however, this needs to be confirmed. In summary, CEUS and TIC are useful in the evaluation of the microcirculation of transplanted kidneys, and can provide reference values for early diagnosis and treatment of DGF. 


\section{ACKNOWLEDGMENTS}

Thank you to all the authors. This study did not receive any funding support.

\section{REFERENCES}

Angelescu M, Kraus T, Wiesel M, Hergesell O, et al. (2003). Assessment of renal graft function by perioperative monitoring of cortical microcirculation in kidney transplantation. Transplantation 75: 1190-1196.

Brier ME, Ray PC and Klein JB (2003). Prediction of delayed renal allograft function using an artificial neural network. Nephrol. Dial. Transplant. 18: 2655-2659.

Barozzi L, Valentino M, Bertolotto M, Pavlica P (2010). Contrast enhanced ultrasound of renal diseases. Arch. Ital. Urol. Androl. 82: 232-237.

Correas JM, Claudon M, Tranquart F and Helenon AO (2006). The kidney: imaging with microbubble contrast agents. Ultrasound Q. 22: 53-66.

Gjertson DW (2000). Impact of delayed graft function and acute rejection on kidney graft survival. Clin. Transpl.467-480.

Huang C, Huang C, Hestin D, Dent PC, et al. (2002). The effect of endothelin antagonists on renal ischaemia-reperfusion injury and the development of acute renal failure in the rat. Nephrol. Dial. Transplant. 17: 1578-1585.

Inman SR, Plott WK, Pomilee RA, Antonelli JA, et al. (2003). Endothelin-receptor blockade mitigates the adverse effect of preretrieval warm ischemia on posttransplantation renal function in rats. Transplantation 75: 1655-1659.

Marcén R, Morales JM, Fernandez-Rodriguez A, Capdevila L, et al. (2010). Long-term graft function changes in kidney transplant recipients. NDT Plus 3: ii2-ii8.

Ojo AO, Wolfe RA, Held PJ, Port FK, et al. (1997). Delayed graft function: risk factors and implications for renal allograft survival. Transplantation 63: 968-974.

Perico N, Cattaneo D, Sayegh MH and Remuzzi G (2004). Delayed graft function in kidney transplantation. Lancet 364: 1814-1827.

Schilling M, Holzinger F, Friess H, Seiler C, et al. (1996). Pathogenesis of delayed kidney graft function: role of endothelin-1, thromboxane B2, and leukotriene B4. Transplant. Proc. 28: 304-305.

Sellers MT, Gallichio MH, Hudson SL, Young CJ, et al. (2000). Improved outcomes in cadaveric renal allografts with pulsatile preservation. Clin. Transplant. 14: 543-549.

Shoskes DA and Halloran PF (1996). Delayed graft function in renal transplantation: etiology, management and long-term significance. J. Urol. 155: 1831-1840.

Sola R, Alarcon A, Jimenez C and Osuna A (2004). The influence of delayed graft function. Nephrol. Dial. Transplant. 19 (Suppl 3): iii32-iii37.

Tapiawala SN, Tinckam KJ, Cardella CJ, Schiff J, et al. (2010). Delayed graft function and the risk for death with a functioning graft. J. Am. Soc. Nephrol. 21: 153-161.

Yarlagadda SG, Coca SG, Garg AX, Doshi M, et al. (2008). Marked variation in the definition and diagnosis of delayed graft function: a systematic review. Nephrol. Dial. Transplant. 23: 2995-3003.

Yarlagadda SG, Coca SG, Formica RN, Jr., Poggio ED, et al. (2009). Association between delayed graft function and allograft and patient survival: a systematic review and meta-analysis. Nephrol. Dial. Transplant. 24: 1039-1047.

Zlotnick DM, Axelrod DA, Chobanian MC, Friedman S, et al. (2010). Non-invasive detection of pulmonary hypertension prior to renal transplantation is a predictor of increased risk for early graft dysfunction. Nephrol. Dial. Transplant. 25: 3090-3096. 